\title{
Une approche clinico-biologique intégrée pour interpréter la pathogénicité des variants du gène de la titine
}

\author{
Aurélien Perrin ${ }^{1}$, Raul Juntas-Morales ${ }^{1,2}$, Mireille Cossée ${ }^{1}$
}

Aurélien Perrin Mireille Cossée Laboratoire de Génétique Moléculaire, EA7402,

Centre Hospitalier Universitaire de

Montpellier, Université de Montpellier, France

Raul Juntas Morales

Laboratoire de Génétique Moléculaire, EA7402,

Centre Hospitalier

Universitaire de

Montpellier, Université de

Montpellier, France

Service de Neurologie.

Centre de référence des

Maladies

Neuromusculaires AOC

(Atlantique-Occitanie-

Caraïbe) Centre

Hospitalier Universitaire

de Montpellier, France

Contact

aurelien.perrin@

ext.inserm.fr
La titine est la plus grande protéine présente dans notre organisme. L'isoforme la plus longue a un poids moléculaire de 4200 kilodaltons. Deux filaments de titine à polarité opposée recouvrent chaque moitié du sarcomère entre le disque $\mathrm{Z}$ et la ligne (ou bande) M. La titine joue un rôle crucial dans le maintien de l'intégrité de la structure du sarcomère par l'intermédiaire d'interactions avec de nombreuses protéines dont la myosine, l'actine, l'alphaactinine, les calpaïnes 1 et 3 , l'obscurine, la téléthonine et bien d'autres [1].

Les titinopathies sont des pathologies en relation avec des altérations de la titine codée par le gène TTN. Le développement des techniques de séquençage à haut débit a permis l'identification d'un nombre important de variants du gène de la titine. Précédemment au séquençage à haut débit, presque uniquement la partie C-terminale était séquencée, ce qui explique que la majorité des phénotypes initialement décrits concerne cette seule partie du gène $T T N$. En raison de l'hétérogénéité clinique, du mode d'hérédité (qui peut être autosomique dominant [AD] ou récessif $\mathrm{AR}]$ ), de la fréquence des variants $T T N$ (1,5-3\% au sein de la population générale) et de l'absence d'outils bioinformatiques fiables pour l'interprétation de leur impact fonctionnel, il est souvent difficile de statuer quant au caractère pathogène des variants identifiés chez les patients. Les mécanismes qui sous-tendent la variabilité phénotypique et du mode d'hérédité des titinopathies sont encore mal connus. Ils impliquent les fonctions structurelles de la titine sur la formation et la stabilité $\mathrm{du}$ sarcomère, ainsi que ses interactions avec d'autres protéines.

En raison de la taille gigantesque de la protéine, peu d'équipes au sein de la communauté internationale, et aucune équipe française en particulier, ne proposait jusqu'ici l'analyse en western-blot (WB) de la titine à des fins diagnostiques. Les laboratoires de génétique moléculaire identifient par séquençage à haut débit, chez des patients atteints de myopathie, avec ou sans cardiomyopathie, un nombre important de variants TTN potentiellement pathogènes. Une analyse clinico-biologique intégrée est importante afin d'évaluer la pathogénicité des variants identifiés. L'approche diagnostique consiste à évaluer, chez des patients suspects de titinopathie, les conséquences des variants TTN sur les transcrits, la protéine et ses interactions protéiques, puis de les corréler aux données familiales et phénotypiques. Une part de ces variants pourrait en effet affecter des domaines d'interaction à des protéines partenaires de la titine. Nous aborderons dans cet article la méthodologie et les différents aspects de la démarche diagnostique de patients suspectés de titinopathie, basée sur une approche intégrée phénotype-hérédité-génotype-transcrits-protéine regroupant :

1) les données familiales ;

2) le recueil complet des données cliniques et paracliniques ;

3) l'analyse exhaustive des données génotypiques ; 4) l'analyse des transcrits (ARNm);

5) l'analyse de la protéine titine en western-blot et éventuellement ses partenaires protéiques

Cette approche intégrée (Figure 1) permet d'améliorer la démarche diagnostique en clinique et d'approfondir les connaissances des mécanismes moléculaires qui sous-tendent la variabilité de l'hérédité et du phénotype.

Les Cahiers de Myologie ont déjà abordé le sujet en 2017 (hors-série $\mathrm{n}^{\circ} 1$ ) dans un article intitulé "Pathologies musculaires liées à la titine, un domaine en émergence" par Ana Ferreiro. et J. Andoni Urtizberea [8]. Cet article résumait de façon claire et détaillée l'historique des titinopathies, leurs modes de transmission, les connaissances en 2017 de l'épidémiologie et des caractéristiques phénotypiques de ces pathologies. Nous aborderons dans le présent article l'apport de chaque élément dans notre démarche intégrée visant à étudier la pathogénicité des variants TTN et à améliorer ainsi le diagnostic des titinopathies (Figure 1).

\section{Données familiales}

Les données familiales et le(s) mode(s) de transmission probable(s), autosomique dominant (AD) ou récessif $(\mathrm{AR})$, sont recueillis par le médecin lors de la consultation. 


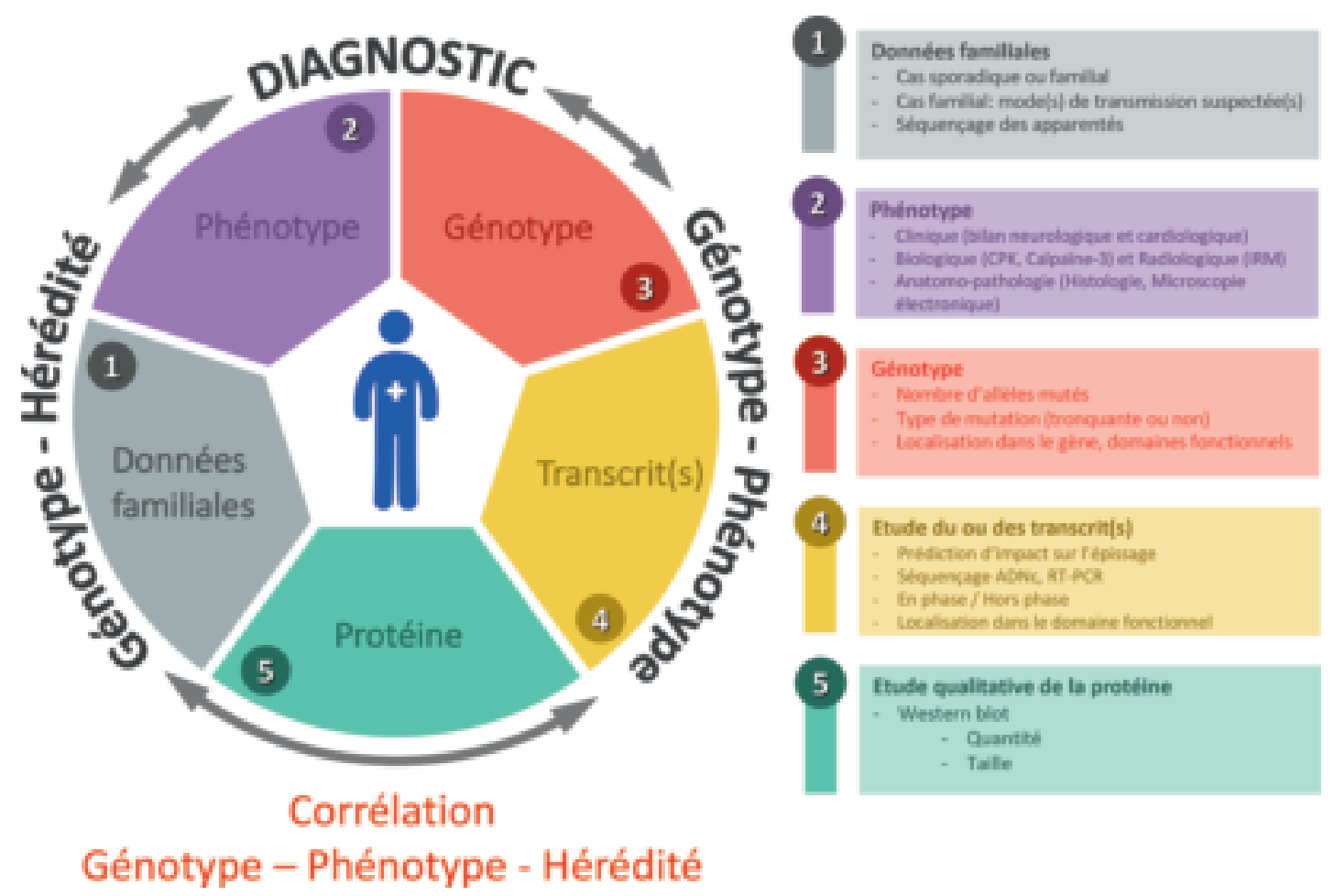

Figure 1

Stratégie d'analyse intégrée des patients suspects de titinopathie.

\section{Données phénotypiques}

Les données phénotypiques (cliniques, histologiques et d'imagerie) des patients sont recueillies par le médecin lors de la consultation (Figure 2). Une analyse phénotypique détaillée est nécessaire étant donné la grande hétérogénéité des phénotypes décrits (voir article "Corrélations phénotype-génotype des titinopathies" par Raul Juntas-Morales et al., de ce même numéro des Cahiers de Myologie) en rapport avec des variants TTN pathogènes. La présence d'anomalies histologiques ou radiologiques évocatrices de titinopathie constitue un élément fondamental lors de l'interprétation des variants.

\section{Clinique}

L'article de ce même numéro des Cahiers de Myologie écrit par le Dr Raul Juntas Morales montre l'étendue des phénotypes décrits et leurs modes de transmission. La description des atteintes peut orienter vers un type de titinopathie pouvant être déjà décrit. Cependant l'émergence de nouveaux patients porteurs de variants potentiellement pathogènes du gène TTN associés à des signes cliniques jusqu'alors non documentés montre que de nouveaux phénotypes sont possibles. Les connaissances actuelles à leur sujet sont encore parcellaires mais en pleine expansion.
Histologie

L'apport de la biopsie musculaire est un élément important dans l'interprétation des variants génétiques du gène TTN. En rapport avec la grande hétérogénéité clinique des patients atteints de titinopathie, de nombreuses lésions histologiques sur les biopsies musculaires ont déjà été décrites. Prises individuellement, la plupart sont peu spécifiques mais le profil histologique d'une combinaison de ces lésions permet souvent d'étayer le diagnostic. Le profil évocateur de titinopathie comprend, en microscopie optique, les anomalies suivantes: une internalisation excessive des noyaux (plus de $10 \%$ et souvent plus de 40-50\%), une prédominance des fibres de type 1, une irrégularité de la taille des fibres, et/ou une présence de minicores. En dehors de ces anomalies communes à la plupart des patients, d'autres plus spécifiques peuvent être présentes dans certaines formes de titinopathies. C'est le cas des corps cytoplasmiques retrouvés chez la plupart des patients atteints de la forme HMERF (" Hereditary Myopathy with Early Respiratory Failure». Dans les formes congénitales décrites par l'équipe d'Ana Ferreiro, la myopathie précoce avec cardiomyopathie létale (EOMFC) [2] et la myopathie à minicores avec cardiomyopathie, des dépôts basophiles en forme d'étoiles peuvent être visualisés et 

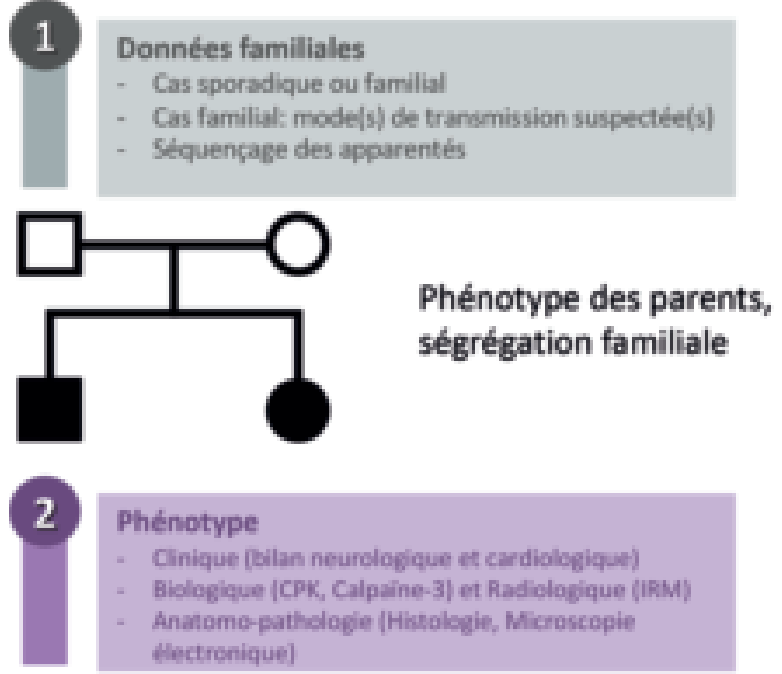

\section{Anatomopathologie}

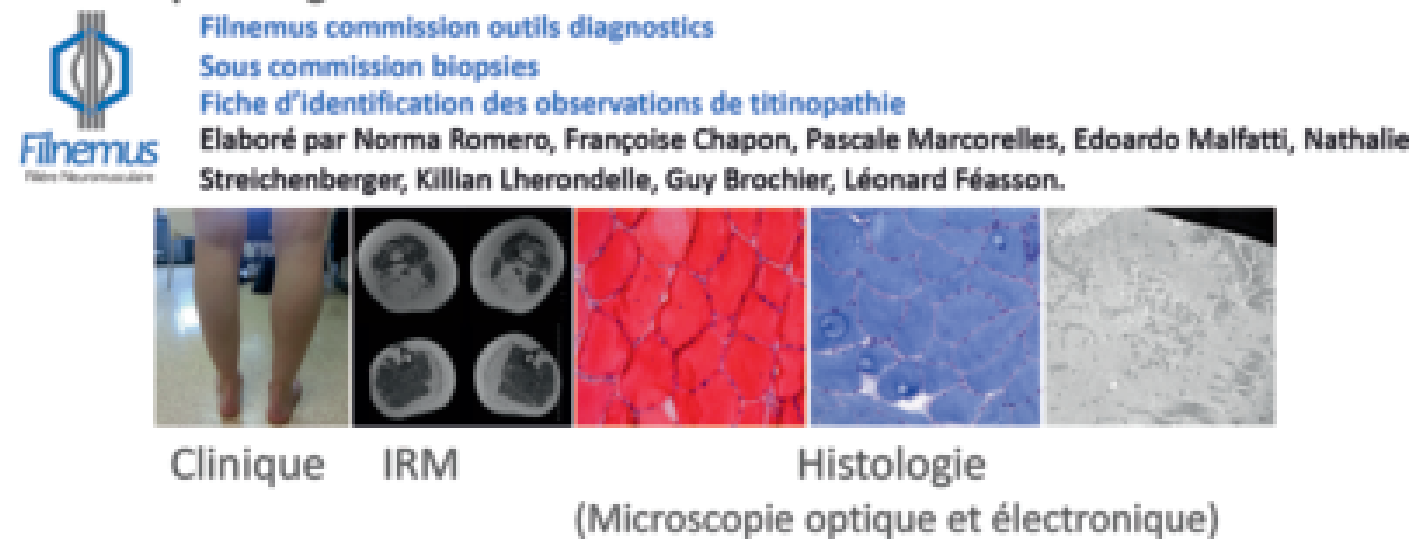

Figure 2

Analyse des données familiales et phénotypiques.

pourraient servir de marqueur histologique de ces formes particulièrement sévères de titinopathie. Des lésions dystrophiques modérées avec nécrose, régénération et fibrose endomysiale accompagnées de vacuoles bordées sont souvent présentes dans les formes dites TMD ("Tibial Muscular Dystrophy") et LGMD2J (" Limb Girdle Muscular Dystrophy 2J / récemment renommée LGMDR10). Des structures de type "casquette" (cap en anglais) ont aussi été décrites dans quelques cas donnant lieu à un tableau de myopathie congénitale. Une désorganisation myofibrillaire et des agrégats de desmine et myotiline peuvent également être présents et visibles en immunohistochimie (Dr. Marcorelles, résultats d'une étude multicentrique dans le cadre de la commission "Outils Diagnostiques " de la filière nationale maladies rares neuromusculaires, Filnemus). En microscopie électronique, il existe surtout des lésions avec désorganisations focales d'une largeur variable et qui concernent habituellement toute la longueur d'un sarcomère.

Imagerie par résonance magnétique (IRM) Compte tenu de l'hétérogénéité phénotypique des titinopathies, il n'existe pas un pattern d'imagerie unique chez les patients concernés. Néanmoins, certains muscles sont plus régulièrement atteints dans certaines formes de titinopathie que dans d'autres. L'exemple le plus frappant est l'involution adipeuse, très évocatrice, du muscle semitendineux au niveau de la loge postérieure de la cuisse, y compris chez des patients ayant des formes distales comme les TMD et HMERF. À un niveau plus distal, les anomalies touchent principalement les muscles releveurs des pieds.

\section{Génotype}

La présence de variant(s) déjà rapporté(s) comme pathogène(s) dans la littérature ou de deux variants 
tronquants en trans chez un patient avec un phénotype clinique compatible permet de poser le diagnostic de titinopathie. En revanche, il est plus difficile de confirmer le rôle pathogène d'un seul variant tronquant devant la présence d'un phénotype évocateur d'une forme rapportée comme AR. Comme le souligne Savarese et al. [3] un deuxième variant peut être localisé dans une région mal couverte par le NGS (mutation située dans une région intronique profonde ou dans une des trois régions répétées du gène TTN). Il est recommandé, dans cette situation, de réaliser une étude de la protéine par WB. Toutefois la mise en place de cette analyse se heurte à des difficultés concernant principalement la taille énorme de la protéine $(3,8 \mathrm{MDa})$ (cf. ci-dessous).

Les variants non tronquants (principalement fauxsens) sont encore plus difficiles à interpréter. Étant donné l'existence d'une grande hétérogénéité clinique avec une expressivité variable aussi bien sur le plan cardiaque que sur le plan musculaire squelettique, les études de ségrégation familiale du variant avec la pathologie peuvent ne pas être informatives. Par ailleurs, les outils de prédictions bioinformatiques sont actuellement peu développés pour l'analyse des variants faux-sens du gène TTN. Pour y remédier, une application en ligne spécifique a été développée récemment, appelé TITINdb [4] (http://fraternalilab.kcl.ac.uk/TITINdb/). Elle intègre les informations de séquence et de structure de la protéine, ainsi que les variants déjà répertoriés comme pathogènes. L'outil permet de visualiser la prédiction des conséquences d'un variant sur la structure de la titine et sur ses interactions avec d'autres protéines, tout en tenant compte des différentes isoformes. Il est également possible d'obtenir des scores de prédictions génétiques comme le score MPA ("MoBiDiC prioritization algorithm") développé dans notre laboratoire et accessible en ligne (https://mobidetails.iurc.montp.inserm.fr/MD/). Il est recommandé de vérifier les prédictions de l'impact des variants TTN sur l'épissage et de les confirmer, si possible, par des études complémentaires au niveau de l'ARN (cf. ci-dessous), ceci apportant un argument supplémentaire de pathogénicité. Un autre aspect à prendre en compte dans l'analyse du génotype est le profil d'épissage des exons du gène TTN. Au cours du développement, les transcrits du gène TTN subissent physiologiquement un épissage alternatif. La forme foetale des transcrits titine reste encore très peu décrite notamment en raison de la difficulté d'accès au matériel biologique foetal. Dans le but de connaitre le degré d'épissage des différents exons au niveau du muscle squelettique, Savarese et al. ont analysé en 2018 les isoformes de la titine par séquençage à haut débit d'ARN (RNAseq) sur des biopsies issues de 42 sujets [5]. Les auteurs ont constaté que certains exons étaient épissés de manière systématique dans les muscles squelettiques après la naissance, tandis que d'autres l'étaient à des degrés variables [5].

Cette cartographie des exons exprimés dans la forme squelettique adulte contribue à la prédiction de l'impact d'un variant TTN.

\section{Analyse des transcrits}

L'analyse des transcrits est essentielle pour évaluer si les ARNm porteurs d'un variant tronquant sont dégradés par le mécanisme de NMD (Nonsens Mediated Decay), système de dégradation des ARNm porteurs d'un codon Stop prématuré), et pour évaluer l'impact des variants d'épissage et des variants faux-sens sur l'épissage (Figure 4). Ces données permettent ainsi de prédire les conséquences au niveau de la protéine, constituant de la sorte des éléments importants pour le diagnostic.

Une des principales difficultés de cette analyse réside dans l'existence d'un épissage alternatif très complexe. Raison pour laquelle il est important de bien connaitre les différentes isoformes de la protéine et leur expression dans les différents tissus afin de pouvoir réaliser une interprétation correcte de la pathogénicité d'un variant [5, 6].

Certains variants rapportés dans la littérature sont localisés dans les exons présents uniquement dans le métatranscrit et absents dans l'isoforme musculaire, N2A. Fernandes-Marmiesse et al. ont rapporté le cas d'un nouveau-né atteint d'un tableau d'arthrogrypose multiple avec hypotonie sévère secondaire à un variant décalant le cadre de lecture (frameshift) homozygote dans l'exon 197 (p.(Lys12887Asnfs"6)). Cet exon n'est pas exprimé dans les différentes isoformes connues en postnatal. Pour les auteurs, les défauts de la protéine en période foetale sont responsables du tableau clinique sévère à la naissance mais la stabilité de la maladie après la naissance serait due à la présence d'une protéine normale exprimée par l'isoforme N2A [7]. Dans le même ordre d'idées, sur les trente patients atteints de titinopathie congénitale de la série récemment publiée par Oates et al. dix avaient des variants pathogènes localisés dans un exon présent uniquement dans le métatranscrit et absents dans l'isoforme N2A [9]. 


\section{Prédictions bioinformatique}

\section{1 \\ MoBidic Prioritization Alzorithm, a Free, Accessible, and Efficlent Fipeline for Single-Nucleotide Variant Mebibic Annotation and Prioritization for Next-Generation Segaencine Routine Molecular Diagnosis

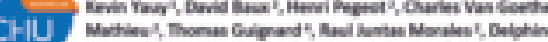

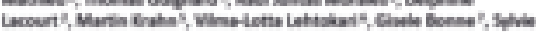

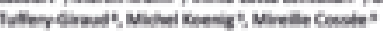

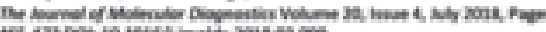

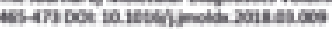 \\ TITindb-a computational tool to assess titin's role as a disease gene

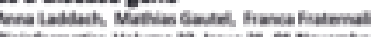

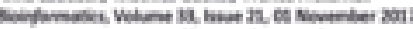

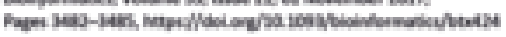

The complexity of tith spliclng pattern in human adult skeletal muscles

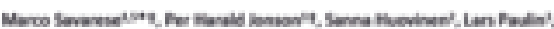

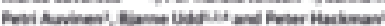

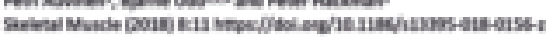

Score MPA httos://mobidetails.iurc.montp.inserm. fr/MD/ (SIFT, PROVEAN, Polyphen-HVAR, Polvphen-HDIV, Mutation Taster, MetaSVM, MetaLR, LRT, FATHMM-MxL, FATHMM

Titin database http://fraternalliab bclac.uk/TIIINdb/ Outils de prediction bast sur la stquence en acides aminés (DUET-mCSM, Condel)

Prediction constquences sur le site d'interaction proteline-protéine (SpPIOCR)

Donntes đexpression (RNAseq), Pexon contenant un variant est-li exprime dans les isoformes musculaires squelettique ou cardiaque adultes?

Allèle 1

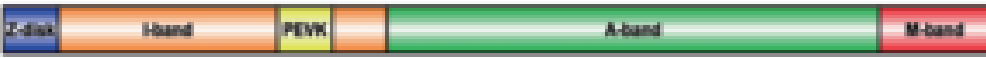

Allèle 2

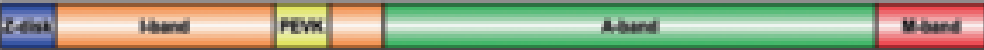

Nombre d'allèles mutes, impact du variant sur la structure, domaine fonctionnel? Type varlant (faux-sens, tronquant) impact sur l'épissage?

Figure 3

Analyse du génotype.

ftude du ou des transcriti(s)

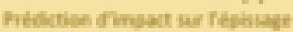

Stquençage Aowk, it pot

In phase / Hors phume

cocatiation dans le donsine fonctienes

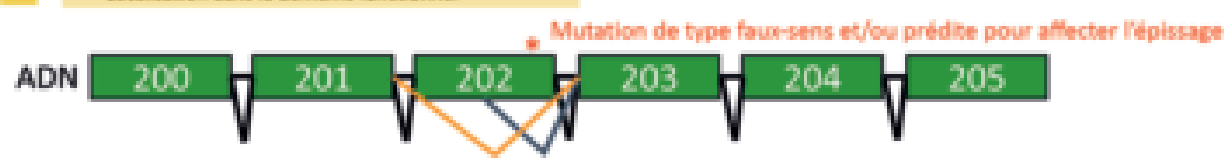

Conséquences potentielles de la mutation :

\begin{tabular}{|c|c|c|c|c|c|c|c|}
\hline ARN & 200 & 201 & 202 & 203 & 204 & 205 & Mas dreflet int repissage \\
\hline RN & 200 & 201 & 202 & 203 & 204 & 205 & Stephase : deletion ofune partie \\
\hline & 200 & 201 & 203 & 204 & 205 & & Saut de Fewon 202 \\
\hline
\end{tabular}

\section{Nombre de populations d’ARNm? Décalage du cadre de lecture? Apparition d'un codon STOP? Dégradation des ARNs anormaux? Expression de l'exon muté dans les muscles atteints?}

Figure 4

Exemple hypothétique d'une étude de transcrit(s) du gène TTN.

\section{Analyse de la protéine}

Un variant décalant le cadre de lecture (variant nonsens, ou frameshift en anglais) aura pour conséquence la synthèse d'une protéine tronquée dont il faudra évaluer si elle est présente ou non dans le muscle du patient. Une variation affectant l'épissage peut aboutir au décalage du cadre de lecture ou à la perte d'un domaine protéique si le cadre de lecture 
est conservé. Les conséquences protéiques des variations de type faux-sens sont actuellement les plus difficiles à évaluer. En effet, le changement d'un acide aminé peut affecter la fonction du domaine dans lequel il est inclus. La titine est composée de nombreux domaines fonctionnels impliqués dans la signalisation musculaire, l'élasticité du muscle, les interactions avec les autres protéines du cytosquelette, l'ancrage à la bande $\mathrm{M}$ ou la bande $\mathrm{Z}$. Il a été mis en évidence à plusieurs reprises une réduction secondaire de la calpaïne-3 dans des muscles de patients porteurs de variants pathogènes dans l'extrémité 3' du gène TTN, ce qui pourrait contribuer à la physiopathologie de la maladie.

L'analyse de la titine est difficile du fait qu'il s'agit d'une protéine géante. Les isoformes de la titine ont un poids moléculaire compris entre 1000 et $3800 \mathrm{kDa}$ alors que pour la majorité des protéines d'une cellule, le poids moléculaire est habituellement de 5 à $300 \mathrm{KDa}$. Les méthodes conventionnelles de western-blot ne sont pas adaptées à l'étude de ce type de protéine géante. Pour analyser la protéine, nous avons mis au point une technique de WB faisant appel à une migration des protéines sur gel d'agarose. Cette technique assez particulière et originale permet ainsi de visualiser et discriminer les différentes isoformes de titine squelettique N2A (3,8 MDa), Novex 3 (1 MDa) ou cardiaque N2B
(3 MDa), N2BA (3,3 MDa) (Figure 5). L'objectif de cette technique est d'évaluer les conséquences des variants TTN sur la taille et la quantité de protéine, et de les comparer aux prédictions génomiques et aux résultats de l'analyse des transcrits. Les limites du WB concernent surtout les variants faux-sens dont les conséquences seront principalement structurales sans modification de taille ou de quantité de titine.

Des stratégies d'études fonctionnelles sont à l'étude dans plusieurs laboratoires de recherche. Elles concernent des approches in vitro, par des tests d'interaction de la titine avec ses partenaires ou encore des approches plus globales à partir de modèles de laboratoires (souris, rat, poisson...). Le développement de tests fonctionnels est un enjeu essentiel pour l'évaluation des conséquences des variants TTN et le diagnostic des titinopathies en clinique.

\section{En conclusion}

L'interprétation des variants du gène TTN nécessite une approche globale et intégrée, allant de l'analyse phénotype jusqu'à l'étude de la protéine. Cette approche, associée à la documentation active des cas de titinopathies, contribue à l'amélioration du diagnostic des patients suspectés de titinopathies. Elle comporte toutefois des limites. L'accès au matériel musculaire n'est pas toujours possible, notam-

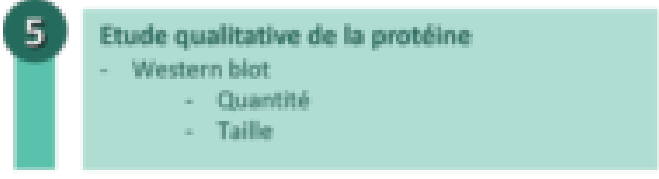

\section{Western blot SDS-Agarose}

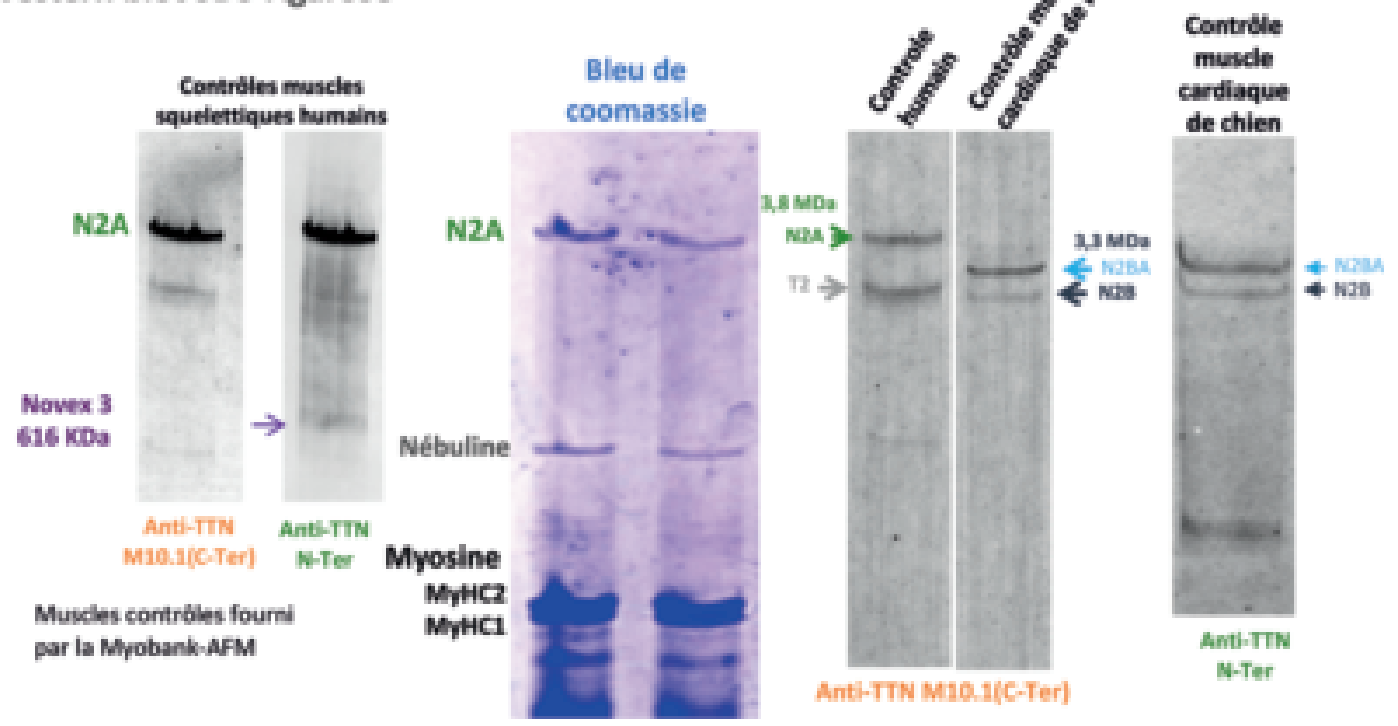

Figure 5

Analyse de la protéine par western blot adapté à l'étude des protéines géantes. 
ment chez les enfants en raison du caractère invasif du prélèvement. Les connaissances scientifiques sur l'expression du gène et les fonctions des différents domaines protéiques ne sont pas encore suffisamment étoffées pour interpréter les conséquences fonctionnelles de la plupart des variants TTN déjà rapportés. L'analyse de l'ensemble des transcrits titine par séquençage à haut débit constitue certainement une technique de choix pour évaluer les conséquences des variants sur les transcrits, du fait de la complexité du pattern d'expression du gène. Cette technique est toutefois coûteuse et fait appel à un traitement bioinformatique des données nécessitant une grande expertise. L'analyse fonctionnelle de la titine est probablement à ce jour le facteur le plus limitant à l'interprétation de la pathogénicité des variants de ce gène. Disposer d'outils d'analyses fonctionnelles comme des modèles animaux serait d'une grande aide, aussi bien pour parfaire l'interprétation des variants que dans un but thérapeutique plus lointain.

An integrated clinical-biological approach for interpreting the pathogenicity of titin gene variants

\section{LIENS D'INTÉRÊT}

Les auteurs déclarent n'avoir aucun lien d'intérêt concernant les données publiées dans cet article.

\section{RÉFÉRENCES}

1. Kontrogianni-Konstantopoulos A, Ackermann MA, Bowman AL, Yap S V., Bloch RJ. Muscle giants: molecular scaffolds in sarcomerogenesis. Physiol Rev 2009; 89 : 1217-67.

2. Carmignac V, Salih MAM, Quijano-Roy S, et al. C-terminal titin deletions cause a novel early-onset myopathy with fatal cardiomyopathy. Ann Neurol 2007 ; 61 : 340-51.

3. Savarese M, Maggi L, Vihola A, et al. Interpreting genetic variants in titin in patients with muscle disorders. JAMA Neurol $2018 ; 75$ : 557-65.

4. Laddach A, Gautel M, Fraternali F. TITINdb-a computational tool to assess titin's role as a disease gene. Bioinformatics 2017 ; 33 : 3482-5.

5. Savarese M, Jonson PH, Huovinen S, Paulin L, Auvinen P, Udd B, Hackman P. The complexity of titin splicing pattern in human adult skeletal muscles. Skelet Muscle 2018; 8 : 11

6. Savarese M, Sarparanta J, Vihola A, Udd B, Hackman P. Increasing role of titin mutations in neuromuscular disorders. $J$ Neuromuscul Dis 2016 ; 3 : 293-308.

7. Fernández-Marmiesse A, Carrascosa-Romero MC, Alfaro Ponce $\mathrm{B}$, et al. Homozygous truncating mutation in prenatally expressed skeletal isoform of TTN gene results in arthrogryposis multiplex congenita and myopathy without cardiac involvement. Neuromuscul Disord 2017 ; 27 : 188-92.

8. Ferreiro A, Urtizberea JA. Pathologies musculaires liées à la titine : un domaine en émergence. Med Sci (Paris) 2017 ; 33 (hors série 1 - Les Cahiers de Myologie) : 16-26.

9. Oates EC, Jones KJ, Donkervoort S, et al. Congenital titinopathy: comprehensive characterization and pathogenic insights. Ann Neurol 2018; 83 : 1105-24.

\section{Retrouvez toutes les Actualités de la Myologie sur les sites de :}

\section{la Société Française de Myologie www.sfmyologie.org}

\section{www. filnemus.fr

la filière de santé neuromusculaire FILNEMUS 